\title{
A Case of Angiolymphoid Hyperplasia with Eosinophilia Aggravated During Pregnancy
}

\author{
Ali Murat Ceyhan', Vahide Baysal Akkaya1, Mehmet Yıldırım¹, Nermin Karahan² \\ ${ }^{1}$ Department of Dermatology, Faculty of Medicine, Süleyman Demirel University, Isparta, Turkey \\ ${ }^{2}$ Department of Pathology, Faculty of Medicine, Süleyman Demirel University, Isparta, Turkey
}

\begin{abstract}
Angiolymphoid hyperplasia with eosinophilia (ALHE) is an uncommon and benign vascular disorder characterized by angiomatous, pink-red papulonodular lesions mainly located on the scalp, neck, and periauricular region. Although the exact cause of ALHE is unknown, several etiologic factors including insect bites or parasites, trauma, hyperestrogen states, and immunologic factors have been implicated in the pathogenesis of the disease. Only a few cases of pregnancy related ALHE so far have been reported in the literature. In this article, we report a case of ALHE with postauricular location aggravated during the first trimester of pregnancy.
\end{abstract}

Key Words: Angiolymphoid hyperplasia with eosinophilia, pregnancy, hormonal factors

Received: 17.08.2009 Accepted: 17.09.2009

\section{Introduction}

Angiolymphoid hyperplasia with eosinophilia (ALHE) is a rare benign vascular tumor characterized by solitary or multiple red to brown papules or nodules found mainly on the head and neck of young adults (1). Although several factors have been implicated in the etiology, the exact causes of ALHE are still unknown (2). There have been only a few reports of pregnancy associated ALHE in the literature (1-4). Herein, we report a case of ALHE with postauricular location aggravated during the first trimester of pregnancy in a young woman.

\section{Case}

A 30-year-old, 12 weeks pregnant woman suffering missed abortion was referred to our clinic due to papulonodular lesions on the right postauricular region associated with marked pruritus. The initial lesion had developed 6 months earlier as a red, elevated, soft papule on the postauricular region. Within the last three months (i.e. the first trimester of pregnancy) the number and size of the lesions had increased with a gradual spread. In addition, the severity of pruritus had significantly increased during this period. The patient did not have a history of oral contraceptive use, insect bite or trauma.

Physical examination revealed numerous, soft, pink-redcoloured papulonodular lesions ranging from $3 \mathrm{~mm}$ to $1 \mathrm{~cm}$ in diameter over the postauricular region of the right ear (Figure 1). The lesions had a tendency to bleed after minor trauma and scratching. The results of the systemic examination were normal, without any evidence of lymphadenopathy.
Laboratory examinations including erythrocyte sedimentation rate, biochemical tests, urine analysis, and complete blood count were within normal limits except for a mildly elevated total lgE level $(298 \mathrm{lU} / \mathrm{ml})$ and HBs-Ag positivity. Absolute eosinophil count in the peripheral blood was within normal limits. Histological examination showed a prominent vascular proliferation of small blood vessels, many of which were lined by endothelial cells with perivascular and interstitial infiltrations composed primarily of lymphocytes and eosinophils (Figure 2). Immunohistochemical staining was positive for CD31; however, direct immunofluorescent histology did not reveal overexpression of estrogen or progesterone receptors in the lesions. Based on these findings, the patient was diagnosed with ALHE and scheduled for cryotherapy at two weeks intervals. The lesions showed partial regression at month 2 of follow-up but the remaining lesion was still quite noticeable.

\section{Discussion}

ALHE was first described by Wells and Whimster in 1969, at which time they employed the term "subcutaneous angiolymphoid hyperplasia" (5). It is characterized by intradermal or subcutaneous, reddish brown, dome-shaped papules and/ or nodules with a relatively regular surface. It mainly occurs in the third to fourth decades of life, with a higher incidence in women. The most common sites are the head and neck, particularly the region around the ears (6). There is controversy regarding whether it is a true vascular neoplasm or a reactive healing process secondary to damage to an artery or vein following a variety of insults. Although the origin of the 


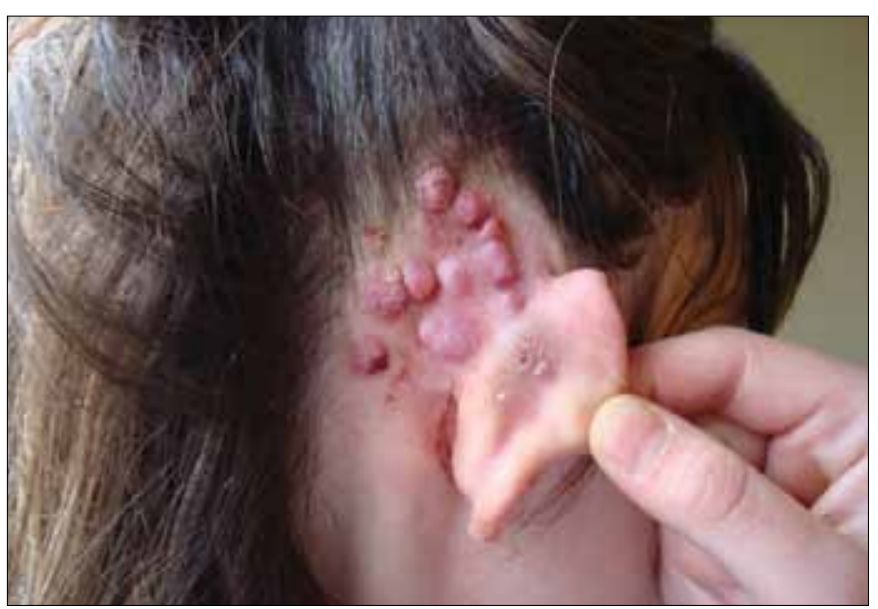

Figure 1. Numerous dome-shaped erythematous soft papules in the postauricular region of the right ear

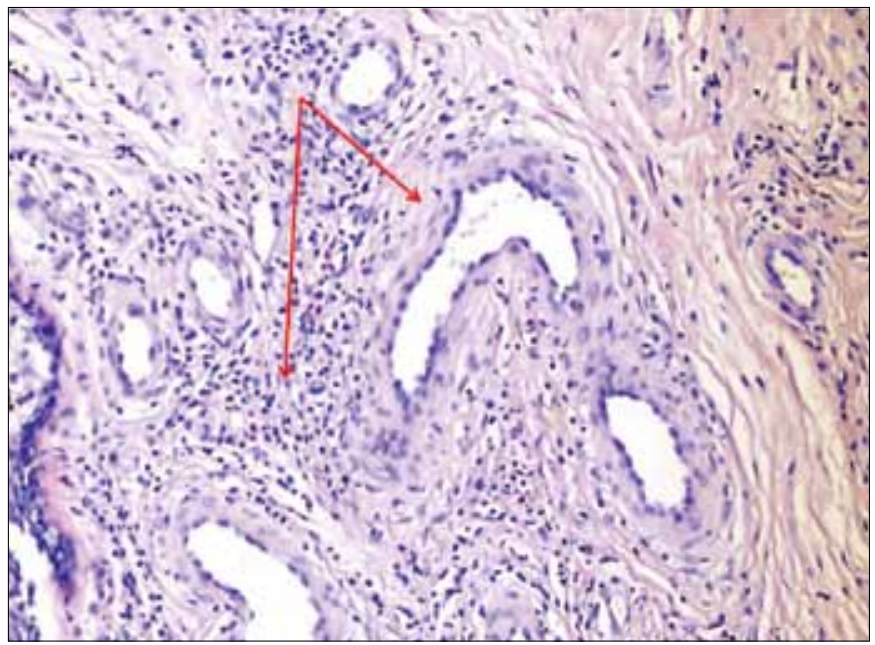

Figure 2. Skin biopsy specimen showing prominent proliferation of small vessels with plump endothelial cells, and perivascular and interstitial infiltration of lymphocytes, histiocytes, and eosinophils (hematoxylin-eosin, original magnification X400)

disease is not exactly known, infections (HTLV or HHV8), hormonal background, trauma, and insect bites have been most commonly suggested as possible etiologic factors (3). Higher prevalence of the disease among women and occurrence of the disease in association with sex hormones are two important observations which indicate that sex hormones may play a role in the pathogenesis of ALHE (6). Pregnancy can also be regarded as a predisposing factor for increased vascular proliferation on the basis of several clinical data suggesting the favouring role of estrogen in ALHE (4). So far, few studies have reported ALHE cases associated with pregnancy (1-4). Olsen et al. reported a series of five ALHE cases associated with pregnancy in their clinicopathologic evaluation of 116 patients. In four out of these five patients, the lesions had occurred for the first time during the first trimester of pregnancy. The fifth patient had a history of multiple asymptomatic lesions for years and their sizes had increased during pregnancy (1). Hollo et al. reported a similar case with an onset of ALHE dur- ing the first trimester of the second pregnancy in a 28-yearold female patient in whom the skin lesion was negative for estrogen and progesterone receptors (3). Zarrin-Khameh et al. described a case of ALHE in a 33-year-old woman who developed an auricular nodule during the second trimester of her pregnancy. They did not show any overexpression of estrogen or progesterone receptors in the lesional skin (2). In another report of pregnancy aggravated ALHE by Moy et al., estrogen and progesterone receptor activity were found to express significant amounts in the lesional skin compared with normal skin (4). In our case, direct immunofluorescent histology did not show overexpression of estrogen or progesterone receptors in the lesional skin.

Pregnancy may also enhance the occurrence or recurrence of lobular capillary hemangioma, including pyogenic granuloma of the gingiva (7). Nichols et al. in a study of twenty-one lobular capillary hemangiomas including lesions from six pregnant patients, demonstrated that all the lesions were negative for estrogen and progesterone receptors. They suggested that estrogen hormone may play a role in the pathogenesis of lobular capillary hemangioma by indirect activity in the absence of estrogen and progesterone receptor expression, but the mechanism of their action still remains unclear (8). In our case, absence of a steroid hormone receptor does not exclude a hormonal role in the aggravation of these lesions. We suggest that elevated levels of estrogens during the first trimester of pregnancy may have an important role in aggravatingf ALHE by an indirect hormonal action.

Spontaneous regression of ALHE rarely occurs. Therefore, treatment is generally necessary. The most common therapeutic options are surgical excision and pulse dye laser. Intralesional injections of glucocorticoids, interferon alfa-2a, and cytotoxic agents, electrodesiccation and cryotherapy have also been reported as therapeutic options with variable levels of success $(2,6)$. Our patient was treated with cryotherapy and only partial regression was observed after 2 months. It is difficult to determine whether this minimal regression is precisely related to the termination of pregnancy. It may also be relevant to the cryotherapy effect as well as spontaneous regression.

The present case of ALHE is of particular interest because the patient's pruritic symptoms and lesions were exacerbated by pregnancy. Although triggering factors such as previous history of oral contraceptive use, trauma, insect bite or infection were not present in our case, aggravation of the disease during the first trimester of pregnancy suggests a potential role of sex hormones in the pathogenesis of ALHE. Thus, we demonstrated a possible hormonal relationship between ALHE and pregnancy, which is also similar to the findings of previous reports.

\section{Conflict of Interest}

No conflict of interest was declared by the authors.

\section{References}

1. Olsen TG, Helwig EB. Angiolymphoid hyperplasia with eosinophilia. A clinicopathologic study of 116 patients. J Am Acad Dermatol 1985;12:781-96. [CrossRef] 
2. Zarrin-Khameh N, Spoden JE, Tran RM. Angiolymphoid hyperplasia with eosinophilia associated with pregnancy: a case report and review of the literature. Arch Pathol Lab Med 2005;129:1168-71.

3. Hollo P, Marschalko M, Sikos G, Harsing J, Horvath A. Angiolymphoid hyperplasia with eosinophilia in pregnancy. J Eur Acad Dermatol Venereol 2005;19:645-6. [CrossRef]

4. Moy RL, Luftman DB, Nguyen $\mathrm{QH}$, Amenta JS. Estrogen receptors and the response to sex hormones in angiolymphoid hyperplasia with eosinophilia. Arch Dermatol 1992;128:825-8. [CrossRef]
5. Wells GC, Whimster IW. Subcutaneous angiolymphoid hyperplasia with eosinophilia. Br J Dermatol 1969;81:1-14. [CrossRef]

6. Akdeniz N, Kösem M, Calka O, Bilgili SG, Metin A, Gelincik I. Intralesional bleomycin for angiolymphoid hyperplasia. Arch Dermatol 2007;143:841-4. [CrossRef]

7. Demir Y, Demir S, Aktepe F. Cutaneous lobular capillary hemangioma induced by pregnancy. J Cutan Pathol 2004;31:77-80. [CrossRef]

8. Nichols GE, Gaffey MJ, Mills SE, Weiss LM. Lobular capillary hemangioma: an immunohistochemical study including steroid hormone receptor status. Am J Clin Pathol 1992;97:770-5. 